\title{
X-ray magnetic circular dichroism study of the blocking process in nanostructured iron-iron oxide core-shell systems
}

\author{
F. Jiménez-Villacorta, ${ }^{1,}{ }^{*}$ C. Prieto, ${ }^{2}$ Y. Huttel,,${ }^{2}$ N. D. Telling, ${ }^{3}$ and G. van der Laan ${ }^{4}$ \\ ${ }^{1}$ Department of Chemical Engineering, Northeastern University, Boston, Massachusetts 02115, USA \\ ${ }^{2}$ Instituto de Ciencia de Materiales de Madrid, CSIC, Cantoblanco, E-28049 Madrid, Spain \\ ${ }^{3}$ Institute for Science and Technology in Medicine (ISTM), Guy Hilton Research Centre, Keele University, Thornburrow Drive, \\ Hartshill, Stoke-on-Trent ST4 7QB, United Kingdom \\ ${ }^{4}$ Diamond Light Source, Chilton, Didcot, Oxfordshire OX11 ODE, United Kingdom \\ (Received 16 March 2011; revised manuscript received 26 October 2011; published 15 November 2011)
}

\begin{abstract}
Experimental evidence for magnetothermal behavior in iron-iron oxide nanostructured systems has been obtained using x-ray absorption spectroscopy (XAS) and x-ray magnetic circular dichroism (XMCD) at the Fe $L_{2,3}$ edges. The purpose of this study is the determination of the blocked state in these spin-glass-like core-shell systems. A first overview of the magnetic species participating in the magnetic response was obtained by analyzing the XMCD at saturating fields. Also, the XAS revealed the existence of an antiferromagnetic FeO phase, likely located at the interface regions. Finally, measurements were performed at low temperature and intermediate field, where a frozen state below the blocking energy is observed. The results show that the oxide phase spins are oriented at low temperature, while the magnetic spins of the metallic core do not contribute to the XMCD, suggesting that the blocking process mainly involves the magnetic particle superspins rather than the oxide coverage phase.
\end{abstract}

DOI: 10.1103/PhysRevB.84.172404

PACS number(s): 75.75.-c, 75.50.Tt, 78.70.Dm

Magnetic core-shell nanoparticles and nanostructures, formed by a ferromagnetic core surrounded by an antiferromagnetic or weakly magnetic oxide shell, are a subject of intense study at both the basic and applied research levels, with an aim to understanding and predicting the magnetic phenomena arising at the interface. ${ }^{1,2}$ This effort has recently been aided by developments in preparation methods, ${ }^{3,4}$ resulting in, e.g., the precise control of the composition of the metal oxide $^{5}$ and the production of compacted metal-oxide granules, in which metal nanoparticles are uniformly embedded in an oxide matrix. Such an enhanced magnetic stability, in terms of increasing coercivity and remanence, as well as the effective decoupling of the magnetic moments of the particles (superspins) with respect to other particles, are provided by the exchange anisotropy, which is due to strong exchange interactions between the core and shell spins. ${ }^{6,7}$ To this end, several experimental ${ }^{8}$ and numerical ${ }^{9,10}$ studies have stressed the importance of the interactions between the metal cores and oxide shells.

Apart from the effects of magnetic interactions between particles and different phases in granular systems, a particular subject of analysis is the study of magnetization reversal and reorientation processes in magnetic nanostructures. For nanogranular systems, it has been proposed that the magnetic properties result from spin canting, which enables the formation of a spin-glass-like phase at the surface of the particles, as reported for oxide ${ }^{11}$ or metal $^{12}$ nanoparticles. This model has been extended to core-shell systems, suggesting a spin-glass-like state and spin-canting features, mostly at low temperatures, attributed to the oxide phase surrounding the metal cores. ${ }^{13,14}$ This frozen state at low temperatures has led to the conclusion that the evolution of magnetothermal curves in Fe-Fe oxide core-shell systems, such as the abrupt increase of magnetization and the variation of magnetization below a maximum, is due to the effects of polarization and reorientation processes of the oxide phase spins. ${ }^{15-17}$ Furthermore, it has been proposed that a de Almeida-Thouless evolution ${ }^{18}$ of the energy barriers, which is a typical feature of canonical spin glasses, can be attributed to the spin-glass-like behavior of the surface spins in magnetic nanoparticle systems. ${ }^{19}$ On the other hand, some interpretations have considered that the blocking and reorientation processes exhibited in magnetothermal curves are associated with the superspin reorientation, ${ }^{20,21,22}$ and that even the de Almeida-Thouless evolution of the energy barriers can be considered to be an effect of interparticle and/or core-shell interactions. ${ }^{23,24}$

In this paper, we present a detailed study, using the microscopic information gained from $\mathrm{x}$-ray magnetic circular dichroism (XMCD), on the magnetic reorientation processes in a transition-metal core-oxide shell system that exhibits spin-glass-like features at low temperatures. It has been reported that sputtering deposition of iron on cooled substrates at temperatures far below room temperature induces a granular structure and thus a granular magnetic behavior in these materials. $^{21,25,26}$ Additionally, thin enough films deposited at $T_{S}=200 \mathrm{~K}$ also exhibit a complex morphology in which the core-shell entities are interconnected forming granular chains. A scanning probe microscopy study confirms the formation of iron cores and an oxide shell covering and separating the metallic clusters. ${ }^{27}$ In contrast with the film deposited at room temperature, samples prepared at these low temperatures exhibit a morphology of interconnected core-shell entities with average dimensions of around $12 \mathrm{~nm}$.

To study the nanostructured iron-iron oxide system, we focused our attention on samples deposited at $T_{S}=200 \mathrm{~K}$ on Si (100) wafers that were subjected to an in situ post-growth oxidation at the deposition temperature by a controlled process consisting of flowing $\mathrm{O}_{2}$ at a pressure of $1 \times 10^{-1}$ mbar. In this way, as reported elsewhere, ${ }^{28}$ the samples display an effective decoupling of the particles forming the chains, which 
is attributed to a physical separation of the particles through the formation of a core-shell metal-oxide structure.

From data reported in Refs. 27 and 28, the effect of the oxide phase is visible from the exchange bias in the magnetization loops at low temperatures $(\sim 500 \mathrm{Oe}$ at $T=10 \mathrm{~K})$. It can be observed that zero-field-cooling (ZFC) curves at intermediate fields exhibit a strong temperature dependence. In that sense, it is observed that the blocking temperature follows a de Almeida-Thouless dependence ${ }^{18}$ on the applied field in the intermediate field range. ${ }^{27}$ In general terms, this blocked state is removed by increasing the energy of the system, either by applying a strong field or by increasing the temperature, although the deblocking and spin reorientation process are likely influenced by a small in-plane anisotropy and ferromagnetic-antiferromagnetic exchange bias stabilization phenomena at low temperature. ${ }^{8,29}$ Moreover, the ZFC curve at $5 \mathrm{kOe}$ shows that, even at low temperatures, there is no observation of any blocking process at such a high field, and thus an almost complete polarization of the magnetic moments along the direction of the field is hypothesized. Independently of the blocking origin, the issue of this work is to determine if the deblocking process of the spins in these core-shell metal-metal oxide systems at intermediate fields consists of the orientation of the cluster superspins, or if it arises from the thermal activation of the disordered oxide phase, acting with a spin-glass-like behavior at low temperatures.

For this purpose, a detailed study using x-ray absorption spectroscopy (XAS) and XMCD at two different temperatures $(25$ and $300 \mathrm{~K})$ and two different magnetic fields ( 1 and $6 \mathrm{kOe}$ ) is shown to provide precise information, not only on the species forming the oxide phase, since usually iron oxide presents a complex form, but also on the orientation and reversal mechanisms of both the metallic clusters and the oxide phase.

XAS and XMCD measurements at the Fe $L_{2,3}$ edges were carried out using the portable octupole magnet system on beamline 5U.1 at the synchrotron radiation source SRS in Daresbury, UK. The XMCD is the difference signal between
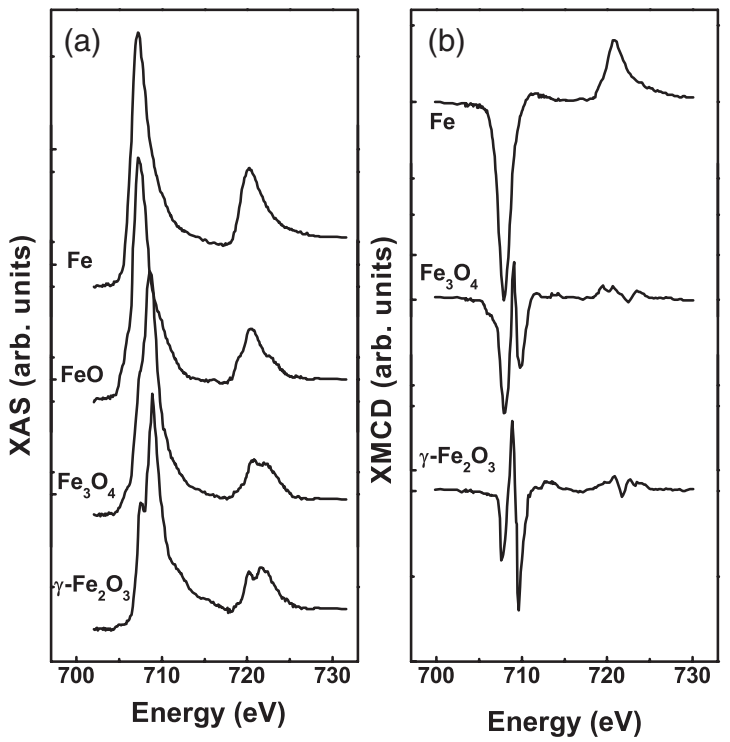

FIG. 1. (a) XAS and (b) XMCD spectra at the Fe $L_{2,3}$ edges of $\mathrm{Fe}$ metal and $\mathrm{Fe}$ oxide reference compounds. the XAS spectra with circular polarization of the $\mathrm{x}$ rays parallel $\left(\sigma^{\uparrow}\right)$ and antiparallel $\left(\sigma^{\downarrow}\right)$ to the applied magnetic field, $\sigma_{\mathrm{XMCD}}=\sigma^{\downarrow}-\sigma^{\uparrow}$. The main purpose of the measurements is to determine the contributions of the different iron species in the magnetization signal, as well as to obtain information about the chemical species that form the oxide shell. ${ }^{30}$ Spectra were collected in total-electron yield (TEY) mode. The helicity of the photons was kept fixed, whereas the applied magnetic field was switched at each data point of the spectrum. In this so called "flipper" mode, the energy shift due to slow beam drifts and monochromator motor movements is minimized between the two opposite magnetized XAS scans. ${ }^{31}$ The angle between the incident $\mathrm{X}$-ray beam and the sample surface was $30^{\circ}$. The XMCD signal, derived from the difference in the XAS spectra for opposite magnetization directions, was analyzed by taking a linear combination of reference iron and iron oxide compounds (displayed in Fig. 1).

Figures 2(a) and 2(b) show the X-ray absorption spectra and dichroic signals, respectively, of the nanostructured sample at room temperature. As a first approximation, we considered that only ferro- and ferrimagnetic phases contribute to the magnitude of the obtained dichroic signal, with their magnetic

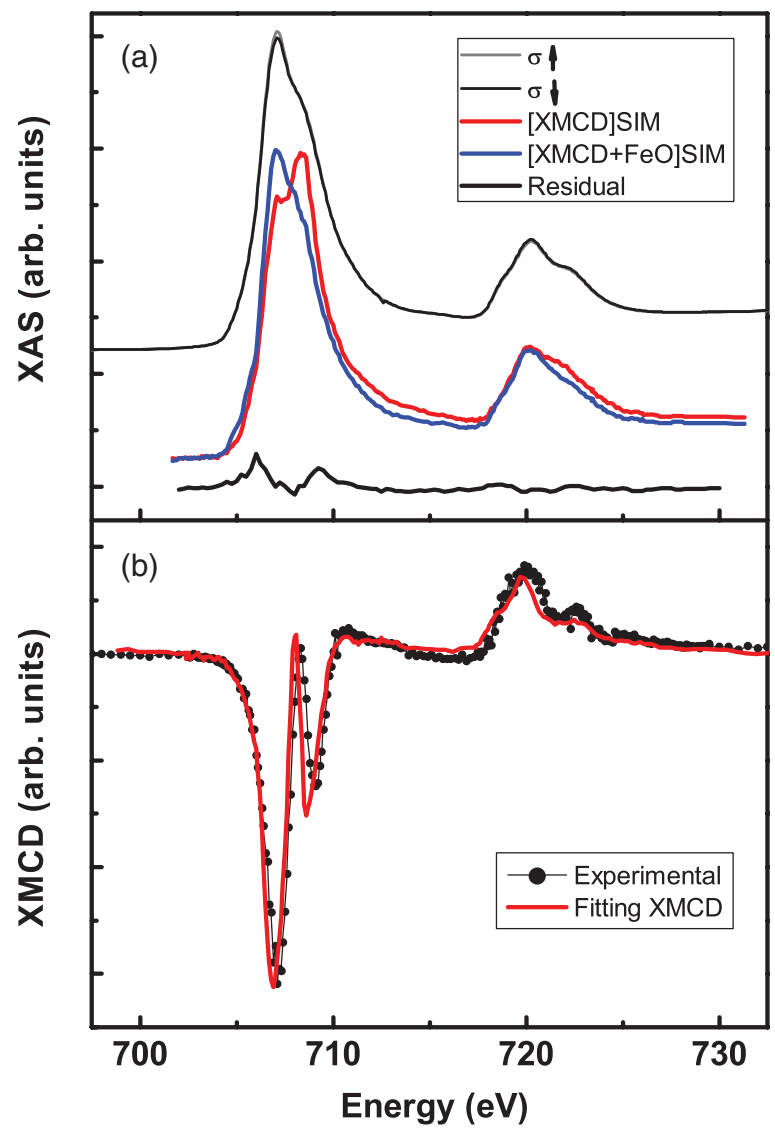

FIG. 2. (Color online) (a) XAS and (b) XMCD spectra at the Fe $L_{2,3}$ edges measured at $T=300 \mathrm{~K}$ and $H=6 \mathrm{kOe}$. The simulations shown in (a) were made using the spectra of the reference compounds taking into account the concentrations obtained from the XMCD ([XMCD] - SIM) and with an added amount of FeO ([XMCD + $\mathrm{FeO}$ ] - SIM). The residual curve gives the difference between the average up and down XAS spectra and the $[\mathrm{XMCD}+\mathrm{FeO}]$ simulation. 
TABLE I. Contributions to the dichroic signals from the different magnetic species at different temperature and applied magnetic field.

\begin{tabular}{lcccc}
\hline \hline & & \multicolumn{3}{c}{ XCMD contribution $(\%)$} \\
\cline { 3 - 5 }$T(\mathrm{~K})$ & $H(\mathrm{Oe})$ & $\alpha-\mathrm{Fe}$ & $\mathrm{Fe}_{3} \mathrm{O}_{4}$ & $\gamma-\mathrm{Fe}_{2} \mathrm{O}_{3}$ \\
\hline 300 & 6000 & $33 \pm 2$ & $53 \pm 2$ & $14 \pm 2$ \\
25 & 6000 & $33 \pm 2$ & $50 \pm 2$ & $17 \pm 2$ \\
25 & 1000 & 0 & $70 \pm 2$ & $30 \pm 2$ \\
\hline \hline
\end{tabular}

response similar to that for the bulk references. Fitting of the dichroic signal by a linear combination using the reference compounds-ferromagnetic $\alpha$-Fe and the ferrimagnetic $\mathrm{Fe}$ oxides $\mathrm{Fe}_{3} \mathrm{O}_{4}$ and $\gamma-\mathrm{Fe}_{2} \mathrm{O}_{3}$ - was performed to quantify the contributions of the different magnetic Fe species in the total XMCD signal: ${ }^{14} \sigma_{\text {fit }}=a \sigma_{\alpha-\mathrm{Fe}}+b \sigma_{\mathrm{Fe}_{3} \mathrm{O}_{4}}+c \sigma_{\gamma-\mathrm{Fe}_{2} \mathrm{O}_{3}}$, where $a, b$, and $c$ are fitting parameters. It should be mentioned that in TEY detection mode, the XAS and XMCD signals are surface-sensitive. Therefore, these are ideal techniques to determine the species forming the oxide shell surrounding the metallic core particles. The result from the analysis at room temperature, displayed in Table I, shows a prominent presence of $\mathrm{Fe}_{3} \mathrm{O}_{4}(53 \% \pm 2 \%)$ as the main component in the oxide phase, together with a small contribution of $\gamma-\mathrm{Fe}_{2} \mathrm{O}_{3}$ of $14 \%$ $\pm 2 \%$. The metallic Fe contribution $(33 \% \pm 2 \%)$ arises from core atoms near the Fe-Fe oxide interface region.

Additional information can be extracted from the XAS spectra. It can be seen in Fig. 2(a) that the experimental spectra do not match the amounts estimated from the XMCD fitting, suggesting the presence of at least another phase that does not contribute to the dichroic signal. The XAS process at the $\mathrm{Fe} L_{2,3}$ edges consists of electric-dipole transitions, primarily $2 p^{6} 3 d^{n} \rightarrow 2 p^{5} 3 d^{n+1}$, from the $2 p$ core levels to empty $3 d$ states. The ground-state electronic configuration, $3 d^{n}$, is split by crystal-field interaction due to the local environment around iron, even determining the high-spin and low-spin states influencing in the XAS features. This effect has been studied in detail in Ref. 32. As a first approximation, it was observed that the $L_{3}$ absorption edge of $\mathrm{Fe}^{2+}\left(O_{h}\right)$ typically exhibits a main peak at a lower energy $(\sim 707 \mathrm{eV})$ followed by a weaker peak or shoulder at a higher energy $(\sim 709 \mathrm{eV}){ }^{33}$ These relative peak intensities are reversed in the case of $\mathrm{Fe}^{3+}$ species. Considering these facts, the octahedral $\mathrm{FeO}$ phase is introduced to simulate the experimental spectra. The best fit is shown in Fig. 2(a). It may be noted that the corresponding residual to evaluate the difference between experimental data and simulation allows the presence of a double peak due to a slight broadening of the sample peak with respect to the calculation from the references. The estimated composition corresponds to $50 \% \pm 2 \% \mathrm{FeO}, 26 \% \pm 2 \%$ $\mathrm{Fe}_{3} \mathrm{O}_{4}, 8 \% \pm 2 \% \gamma-\mathrm{Fe}_{2} \mathrm{O}_{3}$, and $16 \% \pm 2 \% \mathrm{Fe}$, meaning that an important amount of antiferromagnetic $\mathrm{FeO}$, probably located at the interface regions, has to be present in the sample, in agreement with previous works suggesting a partial oxidation and/or reduction process in adjacent metal-oxide interfaces. ${ }^{34}$ In principle, the presence of uncompensated spins in the antiferromagnetic phase would provide an extra signal to the total spectrum, as has been reported for ferromagneticantiferromagnetic systems composed of different atoms. ${ }^{35}$

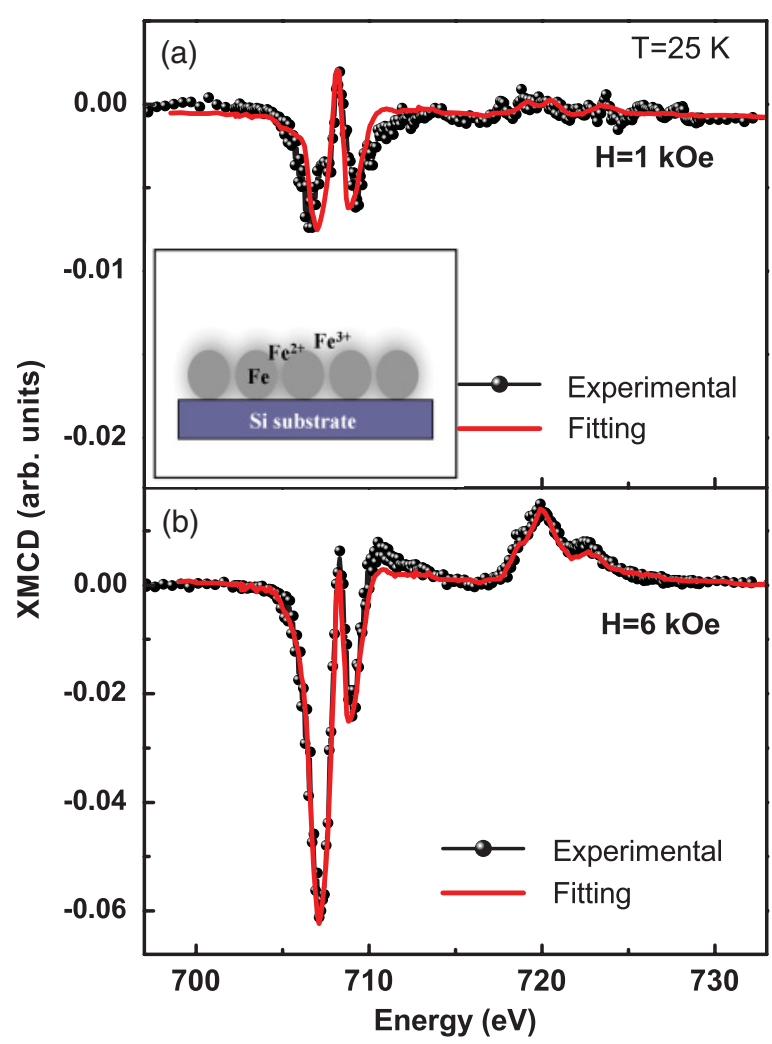

FIG. 3. (Color online) XMCD at $T=25 \mathrm{~K}$ for (a) $H=1 \mathrm{kOe}$ and (b) $H=6 \mathrm{kOe}$. The inset of (a) shows a schematic illustration of the composition and morphology of the iron-iron oxide nanostructured sample.

However, this signal is likely to be very small compared to the ferro- and ferrimagnetic phases observed, and the fitting can be made without considering the contribution of such an uncompensated spin signal.

Another aspect that should be mentioned is that since the TEY detection mode is surface-sensitive, with a sampling depth at the Fe $L$-edge energy in the range of $\sim 6 \mathrm{~nm},{ }^{36}$ and considering that the metallic phase comes from the core, we can estimate an oxide coverage thickness of $\sim 5 \mathrm{~nm}$. Also, considering the oxidation process in transition metals, ${ }^{5,25}$ it should be expected that the oxidation is progressive, and thus the higher oxidation states $\left(\gamma-\mathrm{Fe}_{2} \mathrm{O}_{3}\right)$ are present in the outer regions of the core-shell entity, whereas the lower oxidation state $(\mathrm{FeO})$ is expected to be present at the interface metal-oxide core-shell regions. This model is illustrated in the inset of Fig. 3(a) for the nanostructured granular chain morphology of the sample. Moreover, the presence of an antiferromagnetic compound at low temperatures at the oxide interface regions justifies the remarkable exchange bias values observed. $^{27}$

Summarizing, we determined the species forming the core-shell system, mainly the complex oxide phase, and the compounds that contribute to the magnetic signal at room temperature. Next, our purpose is to investigate the magnetic state at temperatures below the irreversibility point, where a frozen behavior is expected. For this task, we performed XMCD measurements at low temperature $(T=25 \mathrm{~K})$. We have also chosen two different fields: a high field ( $H=6 \mathrm{kOe})$ and an intermediate field $(H=1 \mathrm{kOe})$. 
Figure 3 shows the XMCD spectra at $25 \mathrm{~K}$ using probing fields of 1 and $6 \mathrm{kOe}$. The results of the fitting are included in Table I. From the fit of the dichroic signal at $6 \mathrm{kOe}$ and $25 \mathrm{~K}$, it is observed that the contribution from the oxides and the metallic $\mathrm{Fe}$ to the magnetization changes roughly compared to the room-temperature measurements. The major contribution to the dichroic signal arises from the $\mathrm{Fe}_{3} \mathrm{O}_{4}$. There is also a slight increment in the $\gamma-\mathrm{Fe}_{2} \mathrm{O}_{3}$ contribution, relative to $\mathrm{Fe}_{3} \mathrm{O}_{4}$. This can be attributed to the fact that the $\gamma-\mathrm{Fe}_{2} \mathrm{O}_{3}$ regions are smaller than those in $\mathrm{Fe}_{3} \mathrm{O}_{4}$, with the spins flipping randomly at room temperature, whereas at low temperatures, the magnetization of the ferric spins are along the field direction.

On the other hand, the magnetic signal at $1 \mathrm{kOe}$ and $25 \mathrm{~K}$ comes entirely from the oxide phase. The small signal obtained in the measurement under these conditions hampers an optimum analysis. However, the signal features are sufficiently distinct to extract some conclusions. The best fitting gives $70 \% \mathrm{Fe}_{3} \mathrm{O}_{4}$ and $30 \% \gamma-\mathrm{Fe}_{2} \mathrm{O}_{3}$, while the magnetic contribution from the Fe cores cannot be observed. Also, it is demonstrated that the oxide phase, or at least an important part of it, is polarized and activated under the presence of an intermediate field at very low temperatures. Moreover, the fitting reproduces the intensity of the positive and negative peaks in XMCD that are characteristic of the possible $\mathrm{Fe}$ sites, ${ }^{14,37}$ suggesting that there are no visible effects of spin canting. Finally, it should be noticed that the relative intensities of the two ferromagnetic oxides have changed slightly. The ferric phase exhibits an enhanced contribution to the XMCD signal compared to the mixed-valence magnetite phase. This can be explained considering the amounts obtained when the sample is fully polarized (such as at room temperature and high field). There, the important contribution of $\mathrm{Fe}_{3} \mathrm{O}_{4}$ suggests that the amount of magnetite is larger than the amount of $\gamma-\mathrm{Fe}_{2} \mathrm{O}_{3}$, and thus some parts may remain in the blocked state, although the pinning effect of the antiferromagnetic phase $\mathrm{FeO}$ on the incompletely magnetized $\mathrm{Fe}_{3} \mathrm{O}_{4}$ cannot be neglected.

In conclusion, we have determined the spin activation process in a core-shell iron-iron oxide system, which presents signatures of spin-glass-like behavior at low temperatures, by using x-ray absorption spectroscopy. A combined study of $x$-ray absorption and dichroism reveals the existence of several oxides in the shell region, with a major contribution from $\mathrm{FeO}$ and $\mathrm{Fe}_{3} \mathrm{O}_{4}$. In order to probe different spin states of the system, experiments have been carried out at different fields and temperatures. An analysis of the dichroic signal at the blocked spin-glass-like state reveals that the magnetic contribution comes from the iron oxide coverage spins. Below the blocking temperature in the frozen state, the ferromagnetic clusters do not contribute to the dichroic signal appearing at room temperature and at high fields. Also, no visible signature of a canted spin state has been detected. Hence, we can deduce that the frustrated state in our two-phase iron core-iron oxide shell system, which leads to the spin-glass-like behavior, appears to be related to the magnetic moments of the metallic cores of the granular system.

We are especially thankful to Dr. Eva Céspedes for her invaluable help in the development of the experiment and discussion of the data. *f.jimenezvillacorta@neu.edu

${ }^{1}$ R. C. O'Handley, Modern Magnetic Materials: Principles and Applications (Wiley, New York, 2000).

${ }^{2}$ J. Nogués, J. Sort, V. Langlais, V. Skumryev, S. Suriñach, J. S. Muñoz, and M. D. Baró, Phys. Rep. 422, 65 (2005).

${ }^{3}$ V. Skumryev, S. Stoyanov, Y. Zhang, G. Hadjipanayis, D. Givord, and J. Nogués, Nature (London) 423, 850 (2003).

${ }^{4}$ G. A. Prinz, Science 282, 1660 (1998).

${ }^{5}$ S. Couet, K. Schlage, K. Saksl, and R. Röhlsberger, Phys. Rev. Lett. 101, 056101 (2008).

${ }^{6}$ J. Nogués, V. Skumryev, J. Sort, S. Stoyanov, and D. Givord, Phys. Rev. Lett. 97, 157203 (2006).

${ }^{7}$ J. Nogués and I. K. Schuller, J. Magn. Magn. Mater. 192, 203 (1999).

${ }^{8}$ D. L. Peng, K. Sumiyama, T. Hihara, S. Yamamuro, and T. J. Konno, Phys. Rev. B 61, 3103 (2000).

${ }^{9}$ M. Vasilakaki and K. N. Trohidou, Phys. Rev. B 79, 144402 (2009).

${ }^{10}$ O. Iglesias, A. Labarta, and X. Battle, J. Nanosci. Nanotechnol. 8, 2761 (2008).

${ }^{11}$ R. H. Kodama, A. E. Berkowitz, E. J. McNiff Jr., and S. Foner, Phys. Rev. Lett. 77, 394 (1996).

${ }^{12}$ E. De Biasi, C. A. Ramos, R. D. Zysler, and H. Romero, Phys. Rev. B 65, 144416 (2002).

${ }^{13}$ K. Fauth, E. Goering, G. Schütz, and L. T. Kuhn, J. Appl. Phys. 96, 399 (2004).
${ }^{14}$ L. Signorini, L. Pasquini, F. Boscherini, E. Bonetti, I. Letard, S. Brice-Profeta, and P. Sainctavit, Phys. Rev. B 74, 014426 (2006).

${ }^{15}$ L. del Bianco, D. Fiorani, A. M. Testa, E. Sonetti, L. Savini, and S. Signoretti, Phys. Rev. B 66, 174418 (2002).

${ }^{16}$ E. Winkler, R. D. Zysler, M. Vasquez Mansilla, and D. Fiorani, Phys. Rev. B 72, 132409 (2005).

${ }^{17}$ Q. K.- Ong, A. Wei, and X. M. Lin, Phys. Rev. B 80, 134418 (2009).

${ }^{18}$ J. R. L. de Almeida and D. J. Thouless, J. Phys. A 11, 983 (1978).

${ }^{19}$ H. Wang, T. Zhu, K. Zhao, W. N. Wang, C. S. Wang, Y. J. Wang, and W. S. Zhan, Phys. Rev. B 70, 092409 (2004).

${ }^{20}$ S. A. Koch, G. Palasantzas, T. Vystavel, J. Th. M. De Hosson, C. Binns, and S. Louch, Phys. Rev. B 71, 085410 (2005).

${ }^{21}$ F. Jiménez-Villacorta and C. Prieto, J. Phys.: Condens. Matter 20, 085216 (2008)

${ }^{22}$ M. P. Fernández-García, P. Gorría, J. A. Blanco, A. B. Fuertes, M. Sevilla, R. Boada, J. Chaboy, D. Schmool, and J. M. Grenèche, Phys. Rev. B 81, 094418 (2010).

${ }^{23}$ F. Jiménez-Villacorta, J. Sánchez-Marcos, E. Céspedes, M. García-Hernández, and C. Prieto, Phys. Rev. B 82, 134413 (2010).

${ }^{24}$ A. Kremenovic, B. Antic, V. Spasojevic, M. Vucinic-Vasic, Z. Jaglicic, J. Pirnat, and Z. Trontelj, J. Phys.: Condens. Matter 17, 4285 (2005). 
${ }^{25}$ F. Jiménez-Villacorta, Y. Huttel, A. Muñoz-Martín, C. Ballesteros, E. Román, and C. Prieto, J. Appl. Phys. 101, 113914 (2007).

${ }^{26}$ J. Stankiewicz, F. Jiménez-Villacorta, and C. Prieto, Phys. Rev. B 73, 014429 (2006).

${ }^{27}$ F. Jiménez-Villacorta, C. Munuera, C. Ocal, and C. Prieto, J. Nanopart. Res. 12, 1117 (2010).

${ }^{28}$ F. Jiménez-Villacorta, E. Cespedes, C. Ocal, and C. Prieto, Appl. Phys. Lett. 98, 102513 (2011).

${ }^{29}$ P. S. Normile, J. A. De Toro, T. Muñoz, J. A. González, J. P. Andrés, P. Muñiz, R. E. Galindo, and J. M. Riveiro, Phys. Rev. B 76, 104430 (2007).

${ }^{30}$ R. A. D. Pattrick, G. van der Laan, C. M. B. Henderson, P. Kuiper, E. Dudzik, and D. J. Vaughan, Eur. J. Mineral. 14, 1095 (2002).

${ }^{31}$ E. Dudzik, G. van der Laan, and S. M. Thompson, Synch. Radiat. News 13, 18 (2000).
${ }^{32}$ G. van der Laan and I. W. Kirkman, J. Phys.: Condens. Matter 4, 4189 (1992).

${ }^{33}$ J. P. Crocombette, M. Pollack, F. Mollet, N. Thromat, and M. Gautier-Soyer, Phys. Rev. B 52, 3143 (1995).

${ }^{34}$ T. J. Regan, H. Ohldag, C. Stamm, F. Nolting, J. Lüning, J. Stöhr, and R. L. White, Phys. Rev. B 64, 214422 (2001).

${ }^{35}$ H. Ohldag, A. Scholl, F. Nolting, E. Arenholz, S. Maat, A. T. Young, M. Carey, and J. Stöhr, Phys. Rev. Lett. 91, 017203 (2003).

${ }^{36}$ S. L. M. Schroeder, Solid State Commun. 98, 405 (2010).

${ }^{37}$ T. Kataoka, M. Kobayashi, Y. Sakamoto, G. S. Song, A. Fujimori, F.-H. Chang, H.-J. Lin, D. J. Huang, C. T. Chen, T. Ohkochi, Y. Takeda, T. Okane, Y. Saitoh, H. Yamagami, A. Takana, S. K. Mandal, T. K. Nath, D. Karmakar, and D. Dasgupta, J. Appl. Phys. 107, 033718 (2010). 